\title{
Computational calculations of magnetic relaxation and viscosity in small magnetic grains
}

\author{
J. M. Hernàndez, X. X. Zhang, and J. Tejada \\ Fac. Física, Universitat de Barcelona, Diagonal 647, Barcelona 08028, Spain
}

In this article we present a phenomenological model which simulates very well the magnetic relaxation behavior experimentally observed in small magnetic grains and single domain particles. In this model, the occurrence of quantum tunneling of magnetization below a certain temperature is taken into account. Experimental results for different materials are presented to illustrate the most important behavior deduced from our model. (C) 1996 American Institute of Physics. [S0021-8979(96)79308-5]

The problem of magnetic relaxation is present in many situations of science and technology. In the last years, big efforts have been made with the aim to explain better; (a) the law governing the magnetic relaxation and the effects of energy barrier distributions, ${ }^{1-4}$ (b) the temperature dependence of magnetic viscosity, ${ }^{5-7}$ (c) the relation switching field distribution and magnetic noise in recording media. ${ }^{8}$

The simplest magnetic system for studying the dynamics of magnetization reversal is a single domain particle with uniaxial anisotropy. In the presence of an applied field opposite to the magnetization of the particle, the frequency of the magnetization reversal, at temperature $T$, is given by Ref. 9 $\Gamma=\omega \exp \left(-U / k_{B} T\right)$, where $\omega$ is the attempt frequency at an order of $1 \mathrm{GHz}$, and $k_{B} T$ is the thermal energy. $U$ is the energy barrier height to the magnetization reversal which is in the form of $U=K V\left[1-H / H_{K}\right]^{1 / 2}$ where $K$ is the magnetic anisotropy constant, $V$ is the volume of the magnetic switching unit (assumed to be the entire single domain particle) and $H$ is the applied magnetic field. The anisotropy field, $H_{K}$, is defined in terms of $K$ and the particle saturation magnetization $M_{s}: H_{K}=2 K / M_{s}$.

We also incorporate to our model the effect of quantum tunneling under-barrier transitions of the magnetization below a certain crossover temperature, $T_{\mathrm{CR}},{ }^{10}$ which separates the classical and quantum regimes. Therefore the expression for the attempt frequency can be written as $\Gamma=\omega \exp \left[-U / k_{B} T_{\mathrm{ESC}}(T)\right]$ where the escape temperature,
$T_{\text {ESC }}(T)$, presents in magnetic systems, a temperature dependence characteristic of weak damping processes. Here we assume that $T_{\mathrm{ESC}}(T)=T_{\mathrm{CR}}$ coth $\left(T_{\mathrm{CR}} / T\right)$, because this matches, qualitatively well, the behavior observed in both, magnetic systems and Josephson junctions. ${ }^{11,12}$ In zero field, quantum transitions dominate below $T_{\mathrm{CR}}(0)$. When an external magnetic field is applied, there is a reduction in the value of $T_{\mathrm{CR}}$ which depends on the magnetic field strength, $H$, and the anisotropy field, $H_{K}$ (Refs. 10 and 13)

$$
\begin{aligned}
& T_{\mathrm{CR}}(H)=T_{\mathrm{CR}}(0) \varepsilon^{1 / 2}, \\
& \text { where } \varepsilon=1-H / H_{K} .
\end{aligned}
$$

As one applies a magnetic field $H_{1}$ on such a system consisting of $N$ identical particles, the magnetic moment of the system, at its equilibrium state at temperature $T$, is $\mu_{\text {eq }}\left(H_{1}\right)=N M_{s} V g\left(H_{1}\right)$ with $g(H)=\tanh \left(M_{s} V H / k_{B} T\right)$. If the magnetic field is changed to a new value, $H_{2}$, the evolution of the magnetization moment to a new equilibrium state is governed by

$$
\mu(t)=N V M_{s}\left\{g\left(H_{2}\right)+\left[g\left(H_{1}\right)-g\left(H_{2}\right)\right] \exp (-t \Gamma)\right\} .
$$

If a size distribution of the particles $f(V)$ is taken into account in the model, as it does exist in a real physical material, then the magnetization (density of magnetic moment) relaxes as

$$
M(t)=\frac{\int_{0}^{\infty} f(V) V M_{s}\left\{g\left(H_{2}\right)+\left[g\left(H_{1}\right)-g\left(H_{2}\right)\right] \exp (-t \Gamma(V))\right\} d V}{\int_{0}^{\infty} V f(V) d V} .
$$

In our computation process, it has been assumed that the distribution of volume, $f(V)$, have the form of the so-called log-normal distribution ${ }^{14}$ given by $f(V)$ $=(A / V) \exp \left[-\alpha \log ^{2}\left(V / V_{m}\right)\right]$ where $V_{m}$ is the average volume of the particles and $\alpha$ is related with the inverse of the distribution width.

Equation (3) is the basis of our calculations. This integral has been performed using numerical integration in the interval of volumes in which the distribution of sizes takes significant values.
Figure 1(a) shows the variation with time of the remnant magnetization in zero magnetic field at different temperatures, when previously the system has been saturated by an applied field $H_{1}$, much larger than the anisotropy field, $H_{K}$, and assuming $T_{\mathrm{CR}}=0$. This $M$ vs $t$ data have been fitted using a time-logarithmic law

$$
M(t)=[M(t=0)-M(t \rightarrow \infty)][B-S(T) \log (t)],
$$

where $S(T)$ is the so-called magnetic viscosity, $M(t=0)$ is the initial magnetization and $M(t \rightarrow \infty)$ is the equilibrium 


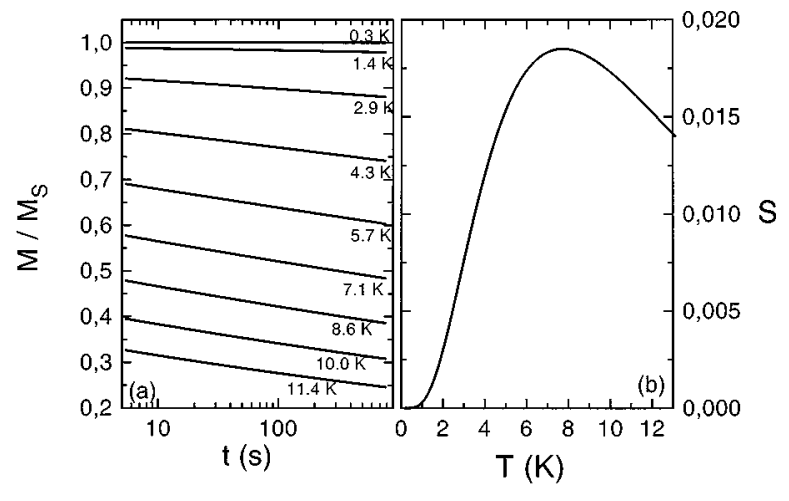

FIG. 1. (a) Relaxation of the magnetization for different temperatures from 0.3 to $11.4 \mathrm{~K}$ as a function of time in a logarithmic scale. (b) Temperature dependence of the magnetic viscosity deduced from (a).

magnetization at the second field, that is $M(t \rightarrow \infty)$ is equal to zero at zero applied field $H_{2}$. From Eq. (4), it is noted that the factor $[M(t=0)-M(t \rightarrow \infty)]$ have all the dependence of the relaxation process on the initial and the final equilibrium states. Therefore, the dependence of $S(T)$ on the temperature is perfectly characterizing the relaxation behavior of the particles.

Figure 1(b) shows the dependence of the magnetic viscosity obtained from Fig. 1(a) on temperature. A near perfect linear dependence of $S$ on $T$ is observed at temperatures much lower than the blocking temperature, which corresponds to the case that the relaxing fraction of the total magnetization is very small. The maximum of the viscosity appears at temperature near the theoretical expectation for the blocking temperature, $T_{B}=K V_{m} /\left[k_{B} \ln \left(\mathrm{t}_{\mathrm{mes}} \omega\right)\right],(7.14 \mathrm{~K}$ in this case) at which the inverse of the jumping frequency, $\Gamma$, for the mean volume particles equals the measuring characteristic time, $t_{\text {mes }}$. At temperatures higher that the blocking, the magnetic viscosity decreases because most of the barriers are overcome in a very short time and do not contribute to the slow relaxation processes.

Quantum tunneling processes are considered by introducing a nonzero value for the crossover temperature $T_{\mathrm{CR}}$, below which the switching processes are independent of temperature. First we have computed the case when the transition temperature $T_{\mathrm{CR}}$ remains constant and the average volume of the grains (and therefore the average barrier height) is different. This situation corresponds to systems having different values for the ratio between the blocking temperature and the crossover temperature, $T_{B} / T_{\mathrm{CR}}$. Both, crossover and blocking temperature scale with the anisotropy constant of the material, but while the crossover temperature does not depend on extensive parameters, blocking is proportional to the volume of the magnetic unit. Therefore the modification of the volume of the particles or grains under the assumption of constancy of the crossover temperature, induces only variations in the blocking temperature. It is, large particles may have large values for the ratio $T_{B} / T_{\mathrm{CR}}$. In Fig. 2 we show different $S(T)$ curves for the situation when the ratio $T_{B} / T_{\mathrm{CR}}$ ranges from 5 to 1 , with $T_{\mathrm{CR}}$ equals $3.3 \mathrm{~K}$. The most remarkable fact is that the maximum of the viscosity disappears when $T_{B} / T_{\mathrm{CR}}$ is 1 . This situation corresponds to the

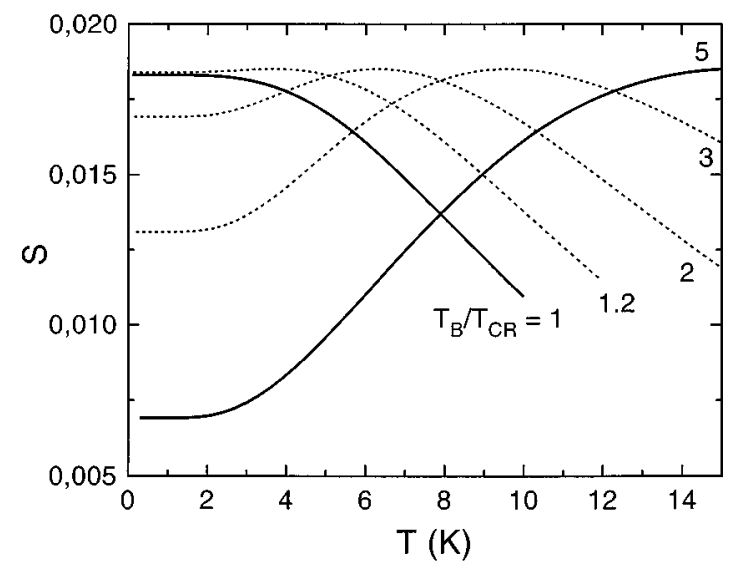

FIG. 2. Effect of the quantum tunneling in the dependence of the viscosity with the temperature for different values of the ratio between the blocking temperature and the crossover temperature.

case when the system enters directly from the classical superparamagnetic regime onto a quantum superparamagnetic regime without any intermediate blocked state. This should be the ideal case to observe the resonance due to quantum coherence. $^{15}$

In Fig. 3 we present the low temperature $S(T)$ values for the case when the applied field, $\mathrm{H}_{2}$, modifies the crossover temperature between the classical and quantum regime, see Eq. (1). This corresponds to the case of a unique set of single domain particles or grains relaxing under the action of different values for the applied field. As the field values increase, the plateau in the viscosity appears at lower temperatures and the quantum viscosity values increase as a consequence of the reduction on the barrier heights.

The situation of Fig. 1 is compared with relaxation experiments performed on a CuCo granular material. Transmission electron patterns in similar systems suggest that this granular material has a log-normal size distribution in agreement with our assumption that the particles in the calculation have a log-normal size distribution. The zero field cooled (ZFC) magnetization vs temperature gives a blocking temperature $T_{B}=8 \mathrm{~K}^{16}$ The relaxation measurements were per-

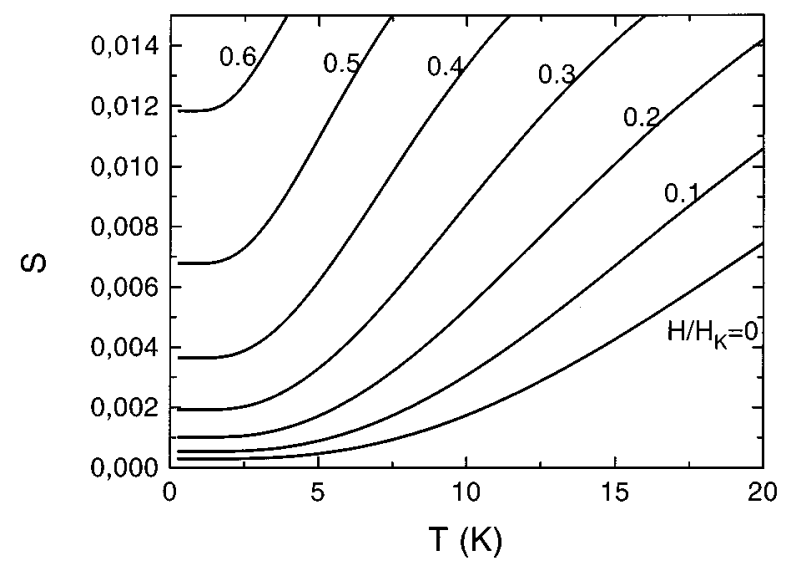

FIG. 3. Temperature dependence of the magnetic viscosity for different applied fields. The numbers near each curve indicate the ratio between the applied field and the anisotropy field. 


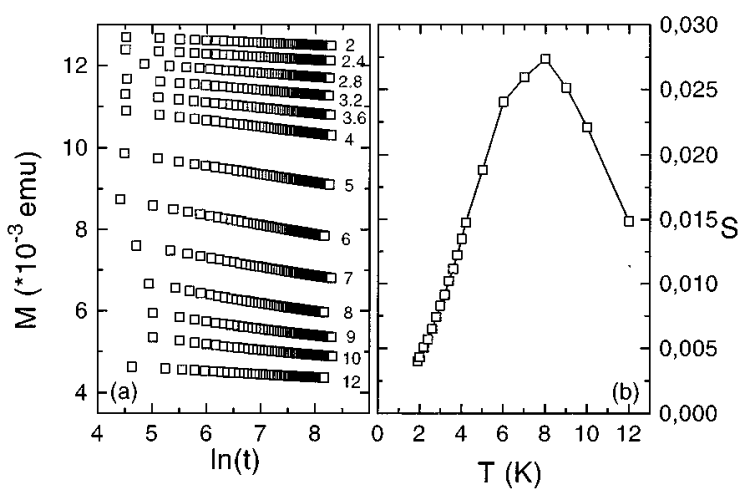

FIG. 4. (a) Magnetic relaxation in a particulate CuCo sample at different temperatures. (b) Dependence of the magnetic viscosity on temperature from (a).

formed from a field $H_{1}=100 \mathrm{Oe}$, after which the field was removed, and then the variation of magnetization with time was measured during few hours. Figure 4(a) shows the relaxation of the remnant magnetization measured at different temperatures; a nearly perfect logarithmic time relaxation behavior is observed. The temperature dependence of magnetic viscosity is presented in Fig. 4(b), and has the same behavior as that shown in Fig. 1(b).

In case of the occurrence of quantum tunneling in the relaxation process, we can compare the experimental results obtained on $\mathrm{CuDy}$ granular material ${ }^{17}$ which has different sizes with the calculated ones presented in Fig. 2. The temperature dependence of magnetic viscosity for samples with composition of $\mathrm{Cu}(100 \AA) \operatorname{Dy}(20 \AA)$ and $\mathrm{Cu}(100 \AA) \operatorname{Dy}(40 \AA)$ is shown in Fig. 5. The two samples have very similar anisotropy fields $H_{K}$ and consequently the same value for the crossover temperature $T_{\mathrm{CR}}$. We have also found, from $\mathrm{ZFC}$ and FC measurements, that these two systems have blocking temperatures of $2.4 \mathrm{~K}$ and $10 \mathrm{~K}$, respectively, which corresponds to the different average size of the grains in the two samples. The theoretical predictions indicate that the transition temperature for these two systems is $T_{\mathrm{CR}}=3 \mathrm{~K}$. There-

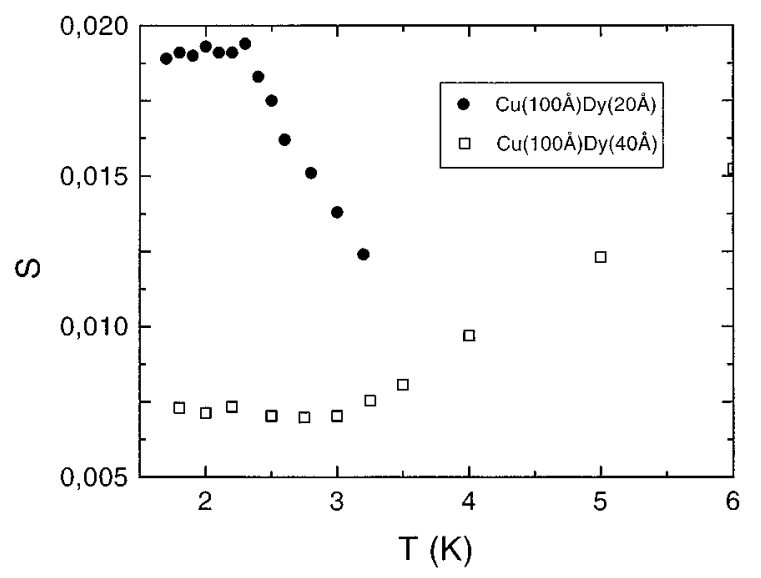

FIG. 5. Magnetic viscosity in two granular structured CuDy thin films: $\mathrm{Cu}(100 \AA)(\mathrm{Dy}(20 \AA)$ (circles) and $\mathrm{Cu}(100 \AA) \mathrm{Dy}(40 \AA)$ (squares).

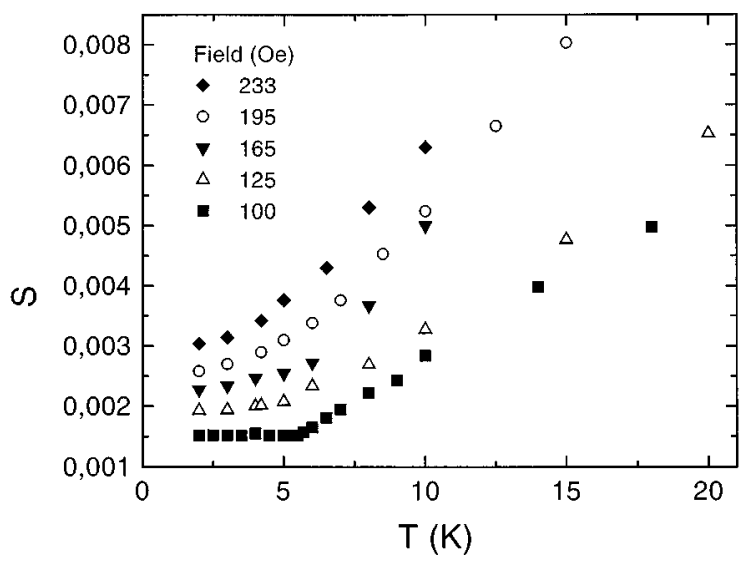

FIG. 6. Magnetic viscosity vs $T$ for different applied fields in a TbFe random magnet.

fore we are in a situation in which we have two different values for the ratio $T_{B} / T_{\mathrm{CR}}, 0.9$ and 3 , respectively. For the sample with $T_{B}=2.4 \mathrm{~K}(\mathrm{Cu}(100 \AA) \mathrm{Dy}(20 \AA))$, we have observed, in agreement with the calculations, that there exists a direct transition from the thermal superparamagnetic behavior to the quantum regime.

In addition, the effect of applied field on the relaxation and quantum tunneling has been found in a $\mathrm{TbFe}$ random magnet. ${ }^{18}$ The dependence of magnetic viscosity on the temperature obtained in relaxation measurements with different applied fields is shown in Fig. 6. It is clearly seen that the behavior of $S(T, H)$ in Fig. 6 is very similar to that in Fig. 3, although the experimental results obtained on the random magnetic thin film corresponds to a relaxation process due to the turning of magnetic moments in different clusters. However, this similarity is not surprising due to the fact that interactions between clusters do not affect the relaxation inside each cluster as a consequence of the large magnetic anisotropy values in these random magnets.

${ }^{1}$ U. Uehara and B. Barbara, J. Phys. (Paris) 47, 235 (1986); D. V Berkov, J. Magn. Magn. Mater. 117, 431 (1992)

${ }^{2}$ J. M. González and J. Tejada, Phys. Rev. B. 49, 3867 (1994).

${ }^{3}$ D. K. Lottis, R. M. White, and E. Dan Dahlberg, Phys. Rev. Lett. 63, 362 (1991).

${ }^{4}$ B. Barbara and L. Gunther, J. Magn. Magn. Mater. 128, 35 (1993).

${ }^{5}$ J. Tejada, X. X. Zhang, and E. M. Chudnovsky, Phys. Rev. B 47, 14977 (1993).

${ }^{6}$ S. H. Charap, J. Appl. Phys. 63, 2054 (1988).

${ }^{7}$ R. W. Chantrell, J. Magn. Magn. Mater. 95, 365 (1991) and references therein.

${ }^{8}$ W. M. Mularie and M. P. Sharrock, J. Appl. Phys. 69, 4938 (1991), and references therein.

${ }^{9}$ L. Neel, Ann. Geophys. 5, 99 (1949).

${ }^{10}$ E. M. Chudnovsky and L. Gunther, Phys. Rev. Lett. 60, 661 (1988).

${ }^{11}$ H. Grabert, P. Olschowski, and U. Weiss, Phys. Rev. B 32, 3348 (1985).

${ }^{12}$ X. X. Zhang, J. Tejada, A. Roig, O. N. Kolov, and E. Molins, J. Magn. Magn. Mater. 137, L235 (1994).

${ }^{13}$ E. Chudnovsky, NATO Workshop Grenoble June 1994.

${ }^{14}$ C. G. Granqvist and R. A. Buhrman, Solid State Commu. 18, 123 (1976).

${ }^{15}$ D. D. Awschalom, J. F. Smyth, G. Grinstein, D. P. Vincenzo, and D. Loss, Phys. Rev. Lett. 68, 3092 (1992).

${ }^{16} \mathrm{X}$. X. Zhang et al. (unpublished).

${ }^{17}$ J. Tejada, X. X. Zhang, and C. Ferrater, Z. Phys. B: Condens. Matter 94, 245 (1994).

${ }^{18}$ J. Tejada, X. X. Zhang, and Ll. Balcells, J. Appl. Phys. 73, 6709 (1993). 